\title{
Representation of Integers as Sums of Fibonacci and Lucas Numbers
}

\author{
Ho Park ${ }^{1}$, Bumkyu Cho ${ }^{1}$, Durkbin Cho ${ }^{1}$, Yung Duk Cho ${ }^{2}$ and Joonsang Park ${ }^{1, *}$ \\ 1 Department of Mathematics, Dongguk University, Seoul 04620, Korea; ph1240@dongguk.edu (H.P.); \\ bam@dongguk.edu (B.C.); durkbin@dongguk.edu (D.C.) \\ 2 Dharma College, Dongguk University, Seoul 04620, Korea; joyd@dongguk.edu \\ * Correspondence: jpark@dgu.edu
}

Received: 14 September 2020; Accepted: 28 September 2020; Published: 1 October 2020

\begin{abstract}
Motivated by the Elementary Problem B-416 in the Fibonacci Quarterly, we show that, given any integers $n$ and $r$ with $n \geq 2$, every positive integer can be expressed as a sum of Fibonacci numbers whose indices are distinct integers not congruent to $r$ modulo $n$. Similar expressions are also dealt with for the case of Lucas numbers. Symmetric and anti-symmetric properties of Fibonacci and Lucas numbers are used in the proofs.
\end{abstract}

Keywords: Fibonacci numbers; Lucas numbers; Zeckendorf's theorem

\section{Introduction}

Let $F_{n}$ denote the $n$th Fibonacci number defined by

$$
F_{0}=0, F_{1}=1, F_{n}=F_{n-1}+F_{n-2} \quad(n \geq 2) .
$$

Lucas numbers $L_{n}$ are defined as $L_{0}=2, L_{1}=1$, and $L_{n}=L_{n-1}+L_{n-2}$ for $n \geq 2$. One can find a lot of properties about Fibonacci and Lucas numbers in any book of Fibonacci numbers (for example, see Koshy's book [1]). Among them, we introduce only a few properties that we need. Binet's formula says that $F_{n}=\frac{1}{\sqrt{5}}\left(\alpha^{n}-\beta^{n}\right)$ for all $n \geq 0$, where $\alpha=\frac{1+\sqrt{5}}{2}$ and $\beta=\frac{1-\sqrt{5}}{2}$ are two roots of the characteristic equation $x^{2}-x-1=0$. Fibonacci numbers of negative indices can be defined in a natural way and Binet's formula also holds true for all $n \in \mathbb{Z}$. Note that $F_{-n}=(-1)^{n+1} F_{n}$ for all $n \in \mathbb{Z}$. Lucas numbers have similar properties such as $L_{n}=\alpha^{n}+\beta^{n}$ and $L_{-n}=(-1)^{n} L_{n}$.

For the following, it is well known that it is possible to represent integers as sums of Fibonacci numbers.

Theorem 1. (Zeckendorf [2]) Every positive integer $m$ can be written uniquely as

$$
m=F_{i_{1}}+F_{i_{2}}+\cdots+F_{i_{s}}
$$

where $i_{s} \geq 2$ and $i_{k}-i_{k+1} \geq 2$ for all $k=1, \ldots, s-1$.

Similarly, every positive integer can be expressed as a sum of nonconsecutive Lucas numbers of nonnegative indices. Note that the uniqueness does not hold for the case of Lucas numbers. For example, $5=L_{0}+L_{2}=L_{1}+L_{3}$. However, the uniqueness holds true if we impose an additional condition that $L_{0}$ and $L_{2}$ must not appear in the expression at the same time [3]. 
The Elementary Problem B-416, proposed by Jakubowski and Hoggatt [4] in 1979, invited readers to show that every positive integer $m$ has at least one representation of the form

$$
m=\sum_{j=-N}^{N} \alpha_{j} F_{j}
$$

with each $\alpha_{j}$ in $\{0,1\}$ and $\alpha_{j}=0$ when $j$ is a multiple of 3 . No solutions of this problem were received at that time and so it was recently presented again by the editor Harris Kwong of the Fibonacci Quarterly in May 2020. Recently, the authors of the present article were able to solve it and sent their solution to the editor by email. It further motivated us to generalize the problem. The purpose of the present article is to give affirmative answers to the generalized problems. More precisely, we show in Theorems 2, 3 and 6 that, given any integers $n$ and $r$ with $n \geq 2$, every positive integer can be expressed as a sum of Fibonacci numbers whose indices are distinct integers not congruent to $r$ modulo $n$. We also obtain similar results about Lucas numbers in Theorems 4, 5 and 7.

Recently, several researchers have applied Zeckendorf's theorem to the Fibonacci codes (see e.g., [5,6]). Since the methods of the proofs in this article are constructive, it would be good if one may exploit our representations to construct new codes.

\section{Even and Odd Expressions in Fibonacci and Lucas Numbers}

If an integer $m$ can be written as

$$
m=\sum_{j=-N}^{N} \alpha_{j} F_{2 j} \quad \text { or } \quad m=\sum_{j=-N}^{N} \alpha_{j} F_{2 j+1}
$$

with each $\alpha_{j}$ in $\{0,1\}$, then we say that $m$ has an even or odd expression in Fibonacci numbers, respectively. The expressions in Lucas numbers are defined analogously.

Theorem 2. Every positive integer has an even expression in Fibonacci numbers.

Proof. By Theorem 1, any positive integer $m$ can be written uniquely as

$$
m=F_{i_{1}}+F_{i_{2}}+\cdots+F_{i_{s}}
$$

where $i_{s} \geq 2$ and $i_{k}-i_{k+1} \geq 2$ for all $k=1, \ldots, s-1$. It will be worth noting that each $i_{k}$ is chosen so that $i_{k} \geq 2$ is the largest integer satisfying

$$
F_{i_{k}} \leq m-F_{i_{1}}-F_{i_{2}}-\cdots-F_{i_{k-1}} .
$$

If $i_{k}$ is odd for some $k$, then we replace $F_{i_{k}}$ by

$$
F_{i_{k}}=F_{i_{k}+1}-F_{i_{k}-1}=F_{i_{k}+1}+F_{-\left(i_{k}-1\right)}
$$

since $F_{-n}=(-1)^{n+1} F_{n}$.

Theorem 3. Every positive integer has an odd expression in Fibonacci numbers.

Proof. For any $m \in \mathbb{N}$, choose the largest integer $i_{1} \geq 0$ such that $F_{2 i_{1}+1} \leq m$. If $m-F_{2 i_{1}+1} \neq 0$, then choose the largest integer $i_{2} \geq 0$ such that $F_{2 i_{2}+1} \leq m-F_{2 i_{1}+1}$. Repeating this process, we arrive at

$$
m=F_{2 i_{1}+1}+F_{2 i_{2}+1}+\cdots+F_{2 i_{s}+1}
$$


where $i_{1} \geq i_{2} \geq \cdots \geq i_{s} \geq 0$. We now show that at most two indices can be the same. Suppose not, i.e., $i_{k}=i_{k+1}=i_{k+2}$ for some $k$. Then, we have

$$
\begin{aligned}
m-\sum_{j=1}^{k-1} F_{2 i_{j}+1} & \geq F_{2 i_{k}+1}+F_{2 i_{k+1}+1}+F_{2 i_{k+2}+1}=3 F_{2 i_{k}+1} \\
& \geq F_{2 i_{k}+1}+F_{2 i_{k}+1}+F_{2 i_{k}}=F_{2\left(i_{k}+1\right)+1}
\end{aligned}
$$

which is a contradiction to the maximality of $i_{k}$. Therefore, $m$ can be written as

$$
m=\sum_{i=0}^{N} \alpha_{i} F_{2 i+1}
$$

where $\alpha_{i}=0,1$, or 2 . If $\alpha_{i}=2$ for some $i$, then we replace $2 F_{2 i+1}$ by

$$
2 F_{2 i+1}=F_{2 i+1}+F_{2 i+1}=F_{2 i+1}+F_{-(2 i+1)} .
$$

It is easy to see that the above properties do not hold for the case of Lucas numbers. For example, 1, 4, 11,29, and 76 do not have even expressions in Lucas numbers and 2, 9, 20,27, and 38 do not have odd expressions in Lucas numbers. However, we can characterize positive integers having even expressions in Lucas numbers as follows.

Theorem 4. Let $m$ be a positive integer. Then, $m$ has an even expression in Lucas numbers if and only if $m$ is not a Lucas number of an odd index.

Proof. Let $m$ be a Lucas number of odd index, say $m=L_{2 n+1}$ for some $n \geq 1$. Suppose $L_{2 n+1}$ has an even expression

$$
L_{2 n+1}=L_{2 i_{1}}+L_{2 i_{2}}+\cdots+L_{2 i_{r}}
$$

in Lucas numbers. Clearly, we have $\left|2 i_{j}\right|<2 n+1$ for all $j$. If $\left|2 i_{j}\right| \leq 2 n-2$ for all $j$, then we obtain a contradiction as

$$
\begin{aligned}
L_{2 n+1} & =\sum_{j=1}^{r} L_{2 i_{j}} \\
& \leq 2 \sum_{k=1}^{n-1} L_{2 k}+L_{0} \\
& =2\left(L_{2 n-1}-L_{1}\right)+L_{0} \\
& =2 L_{2 n-1} \\
& <L_{2 n+1} .
\end{aligned}
$$

Thus, $\left|i_{j}\right|=n$ for some $j$. We may put $i_{1}=n$. Then,

$$
L_{2 n-1}=L_{2 n+1}-L_{2 n}=L_{2 i_{2}}+\cdots+L_{2 i_{r}} .
$$

Repeating the same process by taking $L_{2 n-1}$ instead of $L_{2 n+1}$, we obtain that $L_{1}$ has an even expression in Lucas numbers. This contradicts the fact that 1 does not have an even expression in Lucas numbers.

Suppose that a positive integer $m$ is not a Lucas number of odd index. If $m$ is a Lucas number of even index, we are done. We show by induction on $n$ that, if $m<L_{2 n}$, then $m$ has an even expression in Lucas numbers. We easily check that $5=L_{0}+L_{2}$, and $6=L_{2}+L_{-2}$ for $L_{2}<5,6<L_{4}$. Now, assume that the assertion is true for $n \geq 2$. Then, it is enough to show that every positive integer $m$ with 
$L_{2 n}<m<L_{2(n+1)}$ and $m \neq L_{2 n+1}$ has an even expression. To this end, we consider the following two cases:

Case 1. $L_{2 n}<m \leq 2 L_{2 n}$.

We have $0<m-L_{2 n} \leq L_{2 n}$. If $m-L_{2 n}=L_{2 n}$, then we are done since $m=L_{2 n}+L_{2 n}=$ $L_{2 n}+L_{-2 n}$. If $0<m-L_{2 n}<L_{2 n}$ and if $m-L_{2 n}$ is not a Lucas number of odd index, then by the induction hypothesis $m$ can be expressed as

$$
m=L_{2 n}+L_{2 i_{1}}+\cdots+L_{2 i_{r}}
$$

with $\left|i_{j}\right|<n$ for all $j$. Suppose now that $0<m-L_{2 n}<L_{2 n}$ and that $m-L_{2 n}=L_{2 k+1}$ is a Lucas number of odd index for some $k$. Note that $0 \leq k<n-1$ since $m=L_{2 n+1}$ if $k=n-1$. In this case, $m$ also has an even expression since

$$
\begin{aligned}
m & =L_{2 n}+L_{2 k+1} \\
& =L_{2 n-1}+L_{2 n-2}+L_{2 k+1} \\
& =2 L_{2 n-2}+L_{2 n-3}+L_{2 k+1} \\
& \vdots \\
& =2 L_{2 n-2}+L_{2 n-4}+L_{2 n-6}+\cdots+L_{2 k+2}+2 L_{2 k+1} \\
& =2 L_{2 n-2}+L_{2 n-4}+L_{2 n-6}+\cdots+L_{2 k+2}+2 L_{2 k}+2 L_{2 k-1} \\
& \vdots \\
& =2 L_{2 n-2}+L_{2 n-4}+L_{2 n-6}+\cdots+L_{2 k+2}+2 L_{2 k}+2 L_{2 k-2}+\cdots+2 L_{2}+2 L_{1} \\
& =2 L_{2 n-2}+L_{2 n-4}+L_{2 n-6}+\cdots+L_{2 k+2}+2 L_{2 k}+2 L_{2 k-2}+\cdots+2 L_{2}+L_{0}
\end{aligned}
$$

and $L_{2 k}=L_{-2 k}$ for all $k \in \mathbb{N}$.

Case 2. $2 L_{2 n}<m<L_{2 n+2}$.

Note that $L_{2 n+2}<3 L_{2 n}$ since $L_{2 n+2}=L_{2 n}+L_{2 n+1}=2 L_{2 n}+L_{2 n-1}$. Thus, $2 L_{2 n}<m<$ $L_{2 n+2}<3 L_{2 n}$ and so $L_{2 n}<m-L_{2 n}<2 L_{2 n}$. By the same argument as in Case 1, we can derive our desired assertion.

Theorem 5. Let $m$ be a positive integer. Then, $m$ or $m-2$ has an odd expression in Lucas numbers.

Proof. Any positive integer $m$ can be written as

$$
m=\alpha L_{0}+L_{i_{1}}+L_{i_{2}}+\cdots+L_{i_{s}},
$$

where $\alpha \in\{0,1\}, i_{k+1}-i_{k} \geq 2$ for all $k=1, \ldots, s-1$, and $i_{1} \geq 1$ when $\alpha=0$ and $i_{2} \geq 2$ when $\alpha=1$. If $i_{k}$ is even for some $k=1, \ldots, s$, then we replace $L_{i_{k}}$ by

$$
L_{i_{k}}=L_{i_{k}+1}-L_{i_{k}-1}=L_{i_{k}+1}+L_{-\left(i_{k}-1\right)} .
$$

Therefore, $m-\alpha L_{0}$ has an odd expression if $m-\alpha L_{0}>0$. For $m=2$, we see that $m-2=$ $L_{1}+L_{-1}$.

\section{Representation of Integers beyond Even and Odd Expressions}

In the previous section, we have shown that every positive integer has even and odd expressions in Fibonacci numbers. The ideas underlying their proofs can be developed to prove a more general result. 
Theorem 6. Let $n$ and $r$ be integers with $n \geq 2$. Then, every positive integer $m$ can be expressed as

$$
m=\sum_{j=-N}^{N} \alpha_{j} F_{j}
$$

where $\alpha_{j}=0$ or 1 for all $j$ and $\alpha_{j}=0$ if $j \equiv r(\bmod n)$.

When $n=2$ and $r=0$ or 1 , the theorem reduces to the problem about odd or even expressions for integers. When $n=3$ and $r=0$, it answers the problem B-416.

Proof. Choose the largest integer $j_{1} \geq 2$ such that $F_{j_{1}} \leq m$. Let

$$
i_{1}= \begin{cases}j_{1}-1 & \text { if } j_{1} \equiv r(\bmod n) \text { and } j_{1} \text { is even } \\ j_{1} & \text { otherwise. }\end{cases}
$$

If $m-F_{i_{1}} \neq 0$, then repeat this process by taking $m-F_{i_{1}}$ instead of $m$. Continuing in this manner, we claim that $m$ can be written as

$$
m=F_{i_{1}}+F_{i_{2}}+\cdots+F_{i_{s}}
$$

where

(1) $i_{1} \geq i_{2} \geq \cdots \geq i_{s} \geq 1$,

(2) $i_{k}=j_{k} \Rightarrow i_{k}-i_{k+1} \geq 2$,

(3) $i_{k}=i_{k+1} \Rightarrow i_{k}$ is odd, $i_{k} \equiv r-1(\bmod n)$, and $i_{k+1}-i_{k+2} \geq 2$,

(4) $i_{k} \equiv r(\bmod n) \Rightarrow i_{k}$ is odd, $i_{k}-i_{k+1} \geq 2$, and $i_{k-1}-i_{k} \geq 2$.

Firstly, suppose that these properties hold. According to (3), we see that at most two consecutive indices can be the same. If $i_{k}=i_{k+1}$, then replace $2 F_{i_{k}}$ by

$$
2 F_{i_{k}}=F_{i_{k}}+F_{i_{k}}=F_{i_{k}}+F_{-i_{k}}
$$

where $i_{k} \not \equiv r(\bmod n),-i_{k} \equiv-r+1(\bmod n)$ and $-i_{k}<0$. If $i_{k} \equiv r(\bmod n)$, then replace $F_{i_{k}}$ with

$$
F_{i_{k}}=F_{i_{k}+1}-F_{i_{k}-1}=F_{i_{k}+1}+F_{-\left(i_{k}-1\right)}
$$

where $i_{k}+1 \not \equiv r(\bmod n),-\left(i_{k}-1\right) \equiv-r+1(\bmod n)$ and $-\left(i_{k}-1\right) \leq 0$.

After finishing all the above replacement, $m$ can be written as

$$
m=F_{h_{1}}+F_{h_{2}}+\cdots+F_{h_{t}}+F_{-l_{1}}+F_{-l_{2}}+\cdots+F_{-l_{u^{\prime}}}
$$

where $h_{1}, \ldots, h_{t} \neq \equiv r(\bmod n)$ are distinct positive integers and $-l_{1}, \ldots,-l_{u} \equiv-r+1(\bmod n)$ are distinct nonpositive integers. If $2 r \not \equiv 1(\bmod n)$, then $-r+1 \not \equiv r(\bmod n)$, so we are done. If $2 r \equiv 1$ $(\bmod n)$, then $n \geq 3$ and hence it is enough to replace every term $F_{-l}$ of a nonpositive index by

$$
F_{-l}=F_{-l-1}+F_{-l-2}
$$

since $-l-1,-l-2 \not \equiv r(\bmod n)$. 
Now, it remains to prove (1) to (4). If $i_{k}<i_{k+1}$ for some $k$, then $j_{k} \leq i_{k}+1 \leq i_{k+1} \leq j_{k+1}$ and hence

$$
\begin{aligned}
m_{k-1}:=m-F_{i_{1}}-F_{i_{2}}-\cdots-F_{i_{k-1}} & \geq F_{i_{k}}+F_{j_{k+1}} \\
& \geq F_{j_{k}-1}+F_{j_{k}} \\
& =F_{j_{k}+1},
\end{aligned}
$$

which is a contradiction to the maximality of $j_{k}$. This shows (1).

For the proof of (2), assume that $i_{k}=j_{k}$ and $i_{k}-i_{k+1} \leq 1$ for some $k$. Then, $j_{k}-1=i_{k}-1 \leq$ $i_{k+1} \leq j_{k+1}$, so we get

$$
m_{k-1} \geq F_{i_{k}}+F_{j_{k+1}} \geq F_{j_{k}}+F_{j_{k}-1}=F_{j_{k}+1}
$$

which is also a contradiction.

Now, assume that $i_{k}=i_{k+1}$ for some $k$. Then, $i_{k}=j_{k}-1$ by (2) and the definition of $i_{k}$, and thus $j_{k} \equiv r(\bmod n)$ and $j_{k}$ is even. Thus, $i_{k}$ is odd and $i_{k} \equiv r-1(\bmod n)$. If $i_{k+1}-i_{k+2} \leq 1$, then $j_{k}-2=$ $i_{k}-1=i_{k+1}-1 \leq i_{k+2} \leq j_{k+2}$, so

$$
m_{k-1} \geq F_{i_{k}}+F_{i_{k+1}}+F_{j_{k+2}} \geq F_{j_{k}-1}+F_{j_{k}-1}+F_{j_{k}-2}=F_{j_{k}+1}
$$

which is a contradiction.

Finally, we prove (4). Assume that $i_{k} \equiv r(\bmod n)$ for some $k$. If $i_{k}=j_{k}-1$, then $j_{k} \equiv r(\bmod n)$ and hence $r \equiv r-1(\bmod n)$, which contradicts the condition $n \geq 2$. Thus, $i_{k}=j_{k}$ and $j_{k}$ must be odd. Furthermore, we see that $i_{k}-i_{k+1} \geq 2$ by (2). To obtain $i_{k-1}-i_{k} \geq 2$, suppose, on the contrary, that $i_{k-1}-i_{k} \leq 1$. Then, $0 \leq i_{k-1}-i_{k} \leq 1$ by (1) and $i_{k-1}=j_{k-1}-1$ by $(2)$. Hence, $j_{k-1} \equiv r(\bmod n)$ and $j_{k-1}$ is even. Since $i_{k}$ and $i_{k-1}$ are both odd, we have $i_{k-1}=i_{k}$. However, $i_{k-1}=j_{k-1}-1 \equiv r-1$ $(\bmod n)$. Since $i_{k} \equiv r(\bmod n), i_{k-1}=i_{k}$ leads to a contradiction. This completes the proof.

It turns out that the above theorem also holds for Lucas numbers under the condition $n \geq 3$.

Theorem 7. Let $n$ and $r$ be integers with $n \geq 3$. Then, every positive integer $m$ can be expressed as

$$
m=\sum_{j=-N}^{N} \alpha_{j} L_{j}
$$

where $\alpha_{j}=0$ or 1 for all $j$ and $\alpha_{j}=0$ if $j \equiv r(\bmod n)$.

Proof. For any integer $m \geq 3$ choose the largest integer $j_{1} \geq 2$ such that $L_{j_{1}} \leq m$. Let

$$
i_{1}= \begin{cases}j_{1}-1 & \text { if } j_{1} \equiv r(\bmod n) \text { and } j_{1} \text { is odd } \\ j_{1} & \text { otherwise. }\end{cases}
$$

We repeat this process by taking $m-L_{i_{1}}$ instead of $m$ if $m-L_{i_{1}} \geq 3$. Continuing in this manner, we arrive at

$$
m-L_{i_{1}}-L_{i_{2}}-\cdots-L_{i_{s}} \in\{0,1,2\},
$$


where

$$
\begin{aligned}
& \text { (1) } i_{1} \geq i_{2} \geq \cdots \geq i_{s} \geq 2 \\
& \text { (2) } i_{k}=j_{k} \Rightarrow i_{k}-i_{k+1} \geq 2 \\
& \text { (3) } i_{k}=i_{k+1} \Rightarrow i_{k} \text { is even, } i_{k} \equiv r-1(\bmod n) \text {, and } i_{k+1}-i_{k+2} \geq 2, \\
& \text { (4) } i_{k} \equiv r(\bmod n) \Rightarrow i_{k} \text { is even, } i_{k}-i_{k+1} \geq 2, \text { and } i_{k-1}-i_{k} \geq 2
\end{aligned}
$$

The properties (1) to (4) can be proven by the same argument as in the proof of Theorem 6 , so we omit their proofs. Since $L_{0}=2$ and $L_{1}=1$, we have

$$
m-L_{i_{1}}-L_{i_{2}}-\cdots-L_{i_{s}}=0, L_{1} \text { or } L_{0}
$$

By (3), at most two consecutive indices that occurred on the left-hand side can be the same. If $i_{k}=i_{k+1}$, then replace $2 L_{i_{k}}$ by

$$
2 L_{i_{k}}=L_{i_{k}}+L_{i_{k}}=L_{i_{k}}+L_{-i_{k}}
$$

where $i_{k} \not \equiv r(\bmod n)$ and $-i_{k} \equiv-r+1(\bmod n)$. If $i_{k} \equiv r(\bmod n)$, then replace $L_{i_{k}}$ by

$$
L_{i_{k}}=L_{i_{k}+1}-L_{i_{k}-1}=L_{i_{k}+1}+L_{-\left(i_{k}-1\right)}
$$

where $i_{k}+1 \not \equiv r(\bmod n)$ and $-\left(i_{k}-1\right) \equiv-r+1(\bmod n)$.

After finishing all the above replacement, $m$ can be written as

$$
m=L_{h_{1}}+L_{h_{2}}+\cdots+L_{h_{t}}+L+L_{-l_{1}}+L_{-l_{2}}+\cdots+L_{-l_{u}}
$$

where $h_{1}, \ldots, h_{t} \not \equiv r(\bmod n)$ are distinct positive integers $\geq 2, L \in\{0,1,2\}$ and $-l_{1}, \ldots,-l_{u} \equiv-r+1$ $(\bmod n)$ are distinct negative integers.

If $2 r \equiv 1(\bmod n)$, then $r \not \equiv 0,1(\bmod n)$ and $-r+1 \equiv r(\bmod n)$, so we are done by replacing every term $L_{-l}$ of the negative index by

$$
L_{-l}=L_{-l-1}+L_{-l-2}
$$

since $-l-1,-l-2 \not \equiv r(\bmod n)$.

Now, assume that $2 r \not \equiv 1(\bmod n)$. Since $-r+1 \not \equiv r(\bmod n)$, it is enough to deal with $L=L_{1}$ or $L_{0}$. Let $-l_{1}$ denote the largest negative index. If $r \not \equiv 0,1(\bmod n)$, then we are done. If $r \equiv 1(\bmod n)$, then $-l_{1} \leq-2$ by (3) and (4), so we may replace $L_{1}$ by $L_{1}=L_{0}+L_{-1}$ when $L=L_{1}$. For the case that $r \equiv 0(\bmod n)$, we may assume that $L=L_{0}$. If $-l_{1}=-1$, then $2 \equiv 0(\bmod n)$, which contradicts the condition $n \geq 3$. If $-l_{1}=-2$, then $i_{s-1}=i_{s}=2$ and $n=3$ by (3), so we derive a contradiction as

$$
m-\sum_{k=1}^{s-2} L_{i_{k}}=L_{i_{s-1}}+L_{i_{s}}+L_{0}=8 \geq L_{4} \Longrightarrow j_{s-1}=4 \Longrightarrow i_{s-1}=4
$$

Therefore, $-l_{1} \leq-3$ and we may replace $L_{0}$ by $L_{0}=L_{-1}+L_{-2}$.

Among our future research directions is to extend this analysis to Pell, Pell-Lucas, Jacobsthal and Jacobsthal-Lucas numbers.

Author Contributions: Conceptualization, H.P., B.C., D.C., Y.D.C. and J.P.; methodology, H.P., B.C., D.C., Y.D.C. and J.P. All authors have read and agreed to the published version of the manuscript.

Funding: H.P. was supported by NRF-2019R1C1C1010211. B.C. was supported by NRF-2018R1A2B6001645. D.C. was supported by NRF-2018R1D1A1B07048773. J.P. was supported by the Dongguk University Research Fund of 2018. 
Acknowledgments: The authors would like to express their sincere thanks to the anonymous reviewers for the valuable comments on the manuscript.

Conflicts of Interest: The authors declare no conflict of interest.

\section{References}

1. Koshy, T. Fibonacci and Lucas Numbers with Applications, 2nd ed.; Pure and Applied Mathematics; John Wiley \& Sons: Hoboken, NJ, USA, 2018; Volume 1.

2. Zeckendorf, E. Représentation des nombres naturels par une somme de nombres de Fibonacci ou de nombres de Lucas. Bull. Soc. R. Sci. Liège 1972, 41, 179-182.

3. Brown, J.L. Unique representations of integers as sums of distinct Lucas numbers. Fibonacci Quart. 1969, 7, 243-252.

4. Jakubowski, G.; Hoggatt, V.E. The Elementary Problem B-416. Fibonacci Quart. 1979, 17, 370.

5. Apostolico, A.; Fraenkel, A.S. Robust Transmission of Unbounded Strings Using Fibonacci Representations. IEEE Trans. Inform. Theory 1987, 33, 238-245. [CrossRef]

6. Fraenkel, A.S.; Klein, S.T. Robust Universal Complete Codes for Transmission and Compression. Discr. Appl. Math. 1996, 64, 31-55. [CrossRef]

(c) 2020 by the authors. Licensee MDPI, Basel, Switzerland. This article is an open access article distributed under the terms and conditions of the Creative Commons Attribution (CC BY) license (http:/ / creativecommons.org/licenses/by/4.0/). 